\title{
La construction de la médiation littéraire sur internet : vers un changement de paradigme des pratiques d'écriture
}

The Construction of Literary Mediation on the Internet: Toward a Paradigm

Shift in Writing Practices

Karel Soumagnac

\section{OpenEdition \\ Journals}

Édition électronique

URL : http://journals.openedition.org/edc/796

DOI : 10.4000/edc.796

ISSN : 2101-0366

Éditeur

Université Lille-3

Édition imprimée

Date de publication : 1 décembre 2008

Pagination : 175-188

ISBN : 978-2-917562-00-0

ISSN : 1270-6841

Référence électronique

Karel Soumagnac, « La construction de la médiation littéraire sur internet : vers un changement de paradigme des pratiques d'écriture », Études de communication [En ligne], 31 | 2008, mis en ligne le 01 décembre 2010, consulté le 19 avril 2019. URL : http://journals.openedition.org/edc/796 ; DOI : 10.4000/edc.796

Ce document a été généré automatiquement le 19 avril 2019

(C) Tous droits réservés 


\title{
La construction de la médiation littéraire sur internet : vers un changement de paradigme des pratiques d'écriture
}

\author{
The Construction of Literary Mediation on the Internet: Toward a Paradigm
}

Shift in Writing Practices

Karel Soumagnac

1 L'ambition de ce texte est de comprendre comment s'établit la médiation de la littérature, c'est-à-dire les discours sur la littérature, sur le net. La consultation régulière du réseau nous incite à dire que l'arrivée des outils du web 2.0 a fortement modifié l'organisation des pratiques d'écriture assignées aux « écrits d'écrans » (Souchier, Jeanneret et Le Marec (dir.), 2003 et Jeanneret (dir.), 2005) dans le domaine de l'Internet littéraire. Que deviennent les caractéristiques éditoriales et culturelles de la médiation de la littérature dans ce contexte?

2 L'approche "techno-socio-sémiotique » de la médiation littéraire sur le net permet d'appréhender la complexité de l'espace symbolique littéraire contemporain dans sa dimension numérique ${ }^{1}$. Cette façon d'aborder la médiation a été utilisée pour l'analyse des sites Zazieweb ${ }^{2}$, La Revue des Ressources ${ }^{3}$, Le Matricule des Anges ${ }^{4}$, afin de comprendre le changement de paradigme à l'œuvre dans la construction de l'information littéraire sur le net. Il nous semble en effet que l'écriture de la médiation de la littérature sur le web passe d'un travail axé sur la diffusion de savoirs littéraires à une approche de la littérature centrée sur les pratiques numériques liées à sa consommation. Ainsi, nous allons examiner les modes d'intervention des acteurs sur le web, les formes sémiotiques qu'ils y inscrivent et les pratiques qu'elles suscitent, pour comprendre comment se déploie et s'actualise la médiation de la littérature sur le dispositif Internet. 
3 Tout d'abord, les liens hypertexte rendent compte d'un univers littéraire très médiatisé. Nous chercherons à savoir ensuite si l'organisation éditoriale du site renvoie également à ce processus de médiatisation de l'information littéraire et de l'activité d'écriture des internautes. Nous verrons comment les blogs, en représentant une nouvelle forme de la critique littéraire, y participent. Nous montrerons enfin que l'utilisation des différents médias d'expression sur les sites joue aussi un rôle important dans la redéfinition du cadre socio-technique de la médiation littéraire.

\section{Les liens sortants, un panorama des organes de médiation de la littérature}

4 Les liens hypertexte permettent de connaître l'environnement immédiat des sites, c'està-dire les types d'organes médiatiques, institutionnels, personnels ou associatifs auxquels ils se rattachent. En considérant le site comme un " produit d'information » (Stockinger, 2005), c'est-à-dire un dispositif de diffusion de l'information, notre premier travail d'analyse a donc eu trait à l'étude des liens hypertexte qui partaient des sites. La méthodologie mise en place dans deux études récentes menées sur la médiation littéraire (Jeanneret, 2005) et la littérature numérique (Bouchardon, 2007) a montré la place importante d'une analyse des sites à l'aide d'outils informatiques élaborés pour ces études. Ces outils aident à la compréhension de l'environnement, de la "géographie documentaire » et de la " catégorisation » des sites. L'analyse du voisinage immédiat par une étude manuelle des liens hypertexte peut expliquer, quant à elle, le fonctionnement des pratiques de médiations en terme de « contenus » mais aussi de " formes, d'acteurs et de publics » (Bouchardon, 2007). De cette manière, il est possible de connaître le contexte des sites. Ceux que nous avons étudiés sont au croisement de logiques médiatiques et commerciales. D'une part, les liens hypertexte pointent des ressources documentaires en prise avec des dispositifs de communication médiatique, comme la presse, la radio ou la télévision; c'est le cas notamment avec Zazieweb qui propose systématiquement un lien avec les chaînes de radio et de télévision concernant la médiation du livre ${ }^{5}$ : la communauté autour du web littéraire favorise les organes de production de l'information médiatique en tant que lieux de services et de prestations numériques. D'autre part, il existe une forme de promotion littéraire par voie de marketing. Le fait de favoriser les organes commerciaux incite les publics à consulter des informations traitées principalement par les plateformes de réintermédiation marchande comme le prouve le site Le Matricule des Anges avec les liens vers plusieurs librairies virtuelles ${ }^{6}$.

Ces liens montrent aussi l'organisation du réseau que les sites contribuent à fédérer ou inversement pour lequel ils constituent un maillon fort. En adoptant le point de vue d'Y. Jeanneret et de J. Davallon sur le fait que le «lien» hypertexte matérialise la " réception d'un fait culturel », nous pensons que sa "métasémiotisation " peut aider à comprendre les objectifs de publication des liens que se sont fixés les webmasters. Comme l'ont souligné ces auteurs, le lien «représente» le site vers lequel est dirigé l'internaute. L'ensemble des liens hypertexte donne ainsi à voir un panorama des organes de médiation de la culture littéraire : il n'y a pas un simple "passage » technique d'un site à l'autre mais plutôt une façon de penser l'usage des liens «en termes de situation de réception d'objets culturels » (Jeanneret et Davallon, 2004:51). En fait, les sites offrent une visibilité aux sociabilités qui se créent sur le web en même temps qu'ils les structurent, les dynamisent et les renforcent. Les liens hypertexte sont construits suivant 
une logique de portail. Ils affichent les affinités entre les organes et les acteurs de la médiation de la littérature. Par exemple, les sites créent des liens avec des personnalités connues dans l'édition littéraire et intervenant sur les sites des médias traditionnels. En proposant ces liens, les sites invitent les internautes à consulter des informations sur d'autres sites du domaine. Les liens fonctionnent comme des "traces d'usages" (Davallon, Brochu et Noël-Cadet, in : Souchier, Jeanneret et Le Marec (dir.), 2003), c'est-àdire qu'ils mettent à disposition des internautes des parcours de lecture possible des informations.

6 De plus, il existe un mimétisme dans la construction des liens sur ces sites et ceux propres aux professions spécialistes de la mise en scène de l'information. Les sites littéraires combinent des pratiques éditoriales renvoyant à la fois à la sphère professionnelle de la documentation et à celle du journalisme. Cela a déjà été mis en avant dans des travaux sur les sites d'entreprise qui reprennent les pratiques médiatiques des sites de presse (Cotte, 2007). En ce qui nous concerne, cet emprunt à des sphères professionnelles, relativement étanches les unes des autres, souligne le changement progressif du «territoire» de la médiation littéraire sur le net. Certes, les liens renvoient encore à des sites attachés à la construction de ressources documentaires et fondés sur des bases de données concernant les œuvres, les auteurs, et les critiques d'œuvres par les professionnels de la médiation. Mais il y a de plus en plus une multiplication de liens vers des sites fonctionnant comme des vecteurs d'information médiatique. Les liens hypertexte pointent, nous venons de le voir, les contenus des médias généralistes, mais aussi des sites personnels recensant ce qu'il est possible de trouver actuellement en matière de documentation dans le domaine littéraire : Zazieweb et $L a$ Revue des Ressources proposent ainsi aux publics de se connecter sur des sites experts en littérature dans les domaines de la radio, de la TV et de la presse, sur ceux des revues littéraires, des petits éditeurs, et sur les sites ou blogs de lecteurs amateurs de littérature et d'écrivains.

\section{L'organisation éditoriale des sites, entre fragmentation de l'information et activité d'écriture collective}

7 L'organisation du contenu des sites, en tant que dispositif éditorial, reflète-t-elle également l'objectif de publication de savoirs littéraires informationnels et médiatiques? Nous pensons, comme Bouchardon (Bouchardon, 2007), que les paramètres techniques de la structure éditoriale sont révélateurs d'un positionnement sur le web en terme de projet éditorial et de pratique de l'écriture. Pour comprendre l'organisation éditoriale du site, il s'agit de saisir sa "nature ", son «interface ", «sa structuration » et les " dispositifs de communication » qui lui sont associés. En effet, la particularité du web 2.0 est de proposer de nombreux outils d'édition, de circulation et de réception de l'information. Dans cette perspective, la forme organisationnelle des dispositifs devient intéressante à examiner parce qu'elle donne à voir les conditions de mise en forme des outils de gestion et d'accès à l'information. Internet, avec ses différentes « applications ", ses produits et ses services, ne fonctionne plus de manière unidirectionnelle, ni suivant une « logique de stockage ». Il n'est plus un simple «dispositif de diffusion » mais un média au sens premier du terme permettant une « logique de flux » parce qu'il est dorénavant un "dispositif d'échange » (Clément, in: Bouchardon, 2007 : 13). Les pages d'accueil offrent un bon exemple de ce type de dispositif: sur les trois sites, les espaces d'information sont fragmentés en différentes zones d'édition reliées à l'actualité et à l'activité économique du livre, de 
l'édition et de la librairie ${ }^{7}$. Ainsi, le dispositif éditorial assure une forme de régulation de l'information en proposant de manière systématique le livre comme un objet éditorial, marchand et médiatique.

En même temps, « La description d'un site web [...] intègre [...] la façon dont se définissent les rôles, les acteurs et éventuellement qui ils sont à travers les informations explicitement affichées sur l'organisation du groupe mais aussi à travers les fonctionnalités associées au site. Ces indices permettent souvent de comprendre, au-delà de l'architecture des documents et des fonctionnalités du site, l'activité collective et, en quelque sorte, la « raison sociale » des acteurs sur le web» (Bouchardon, 2007 : 77). Dans cette perspective, chaque site web peut être vu comme une forme organisationnelle de pratiques d'écriture d'acteurs (Chapelain et Loneux, 2007) car il est autant un espace d'information qu'un espace social. Ces acteurs se mobilisent sous la forme de communautés capables de diffuser et, inversement, de s'approprier des objets de savoirs littéraires médiatisés par le web.

Toutefois, les outils du web 2.0 bouleversent l'organisation des dispositifs de soumission des textes, de leur validation, de l'acceptation des membres au sein d'une communauté de médiateurs. Il y a une redistribution des rôles des responsables dans la chaîne d'édition (Bouchardon, 2007, Jeanneret, 2005). C'est le cas avec le site Zazieweb pour lequel la créatrice Isabelle Aveline, en plus de son travail de rédactrice, a un rôle de médiatrice et de modératrice des messages postés sur le site. Les internautes ont la possibilité de déposer et de commenter des informations (Bouchardon, 2007 : 127). Ils légitiment de fait certains auteurs, ouvrages et événements culturels. Les logiques de diffusion n'appartiennent donc plus seulement aux professionnels de la médiation et ne passent plus forcément par des canaux institutionnalisés. Les internautes se trouvent également dans la capacité de produire et de diffuser les mêmes informations que les professionnels dans un contexte où sont utilisés des logiciels d'écriture de grande diffusion: "On assiste [...] à une sorte de déplacement des lieux de pouvoir et des prises sur la culture [...]. Les acteurs traditionnels de la culture tendent à être dépossédés de l'ordre du texte, au bénéfice de ceux qui, pour des raisons de compétence technique ou de puissance économique, sont en position de créer pour les autres les conditions de leur expression " (Jeanneret et Souchier, $2005: 14$ ). De sorte que le processus de production/diffusion est transformé : nous retrouvons, comme dans d'autres domaines, un mode de coopération entre les acteurs pour produire des savoirs. Il existe même un désir de la part du médiateur et de celle de l'usager, de jouer dans l'anticipation de la production du site, les producteurs créant les « conditions » d'« expression » des usagers. La Revue des Ressources a une rubrique consacrée à la création littéraire sur laquelle des écrivains et artistes peuvent venir s'exprimer. Zazieweb, de son côté, met en place des espaces éditoriaux pouvant recueillir les adresses des sites et blogs qui participent à la médiation de la littérature. Les internautes ne sont plus simplement dans la réactivité mais dans la prise de conscience d'une action de coopération pour participer à la construction du site. Il y a ainsi un partage de pouvoir de la fonction éditoriale, et une mise en visibilité de leur activité d'écriture. Nous ne sommes pas dans un modèle horizontal de production des connaissances comme dans le cas de wikipedia mais dans des domaines intermédiaires où l'expert accepte de ne plus être seul maitre d'œuvre. Les utilisateurs contribuent à l'enrichissement collectif des sites et l'ensemble des contenus et services permettent un travail collaboratif. En effet, le travail du professionnel subsiste, et la collaboration de 
l'internaute, lorsqu'elle est retenue, est réintégrée, parce qu'elle a une raison d'être: l'élaboration de la critique littéraire.

\section{Le blog comme nouvelle forme de la critique littéraire}

10 Les formes textuelles de la critique se retrouvent inscrites depuis peu sur les blogs. Ces nouveaux dispositifs d'autopublication constituent une nouvelle façon de construire, de présenter la critique littéraire et de la faire circuler sur le web. Tout d'abord, ils font des espaces d'écriture des lieux d'expression publique et citoyenne où l'information littéraire devient une information d'opinion. Ce constat a déjà été établi dans le domaine journalistique (Cardon et alii, 2006). Sur les sites littéraires, la création de blogs correspond à un acte marquant dans l'actualité, comme cela a été le cas pour le site Le Matricule des Anges avec le «déballage médiatique » sur les Présidentielles de $2007^{8}$. Le format de publication de l'information devient hybride parce qu'il renvoie à la fois à la fonction de publication et à celle de communication. Ce mélange des genres n'est pas nouveau: il a déjà été évoqué dans des analyses de la communication de journaux culturels pour lesquels le genre journalistique est utilisé pour la promotion des objets culturels (Cavelier-Croissant et alii, 2004). Sur le net littéraire, le blog participe à la construction de l'information éditoriale, le contenu intégrant un dispositif qui fait figure de modernité dans la pratique de la médiation de la littérature. Pour autant, il ne s'agit pas seulement de voir le blog comme un outil répondant au culte de la nouveauté dans la démocratisation de l'accès à l'écriture. C'est un dispositif de publication jouant un rôle important dans la diffusion de la critique : à la différence du forum qui assurait la mise en place d'une communauté d'acteurs et la réception des discours, le blog instaure la présence de la critique littéraire sur le net de manière minimaliste et actualisante.

11 C'est pour la même raison économique qu'est envisagée la mise en place des fils RSS (Really Simple Syndication) ${ }^{9}$ et dont il faut distinguer plusieurs niveaux d'utilité ${ }^{10}$. Sur les sites étudiés, cette technologie est un outil de veille documentaire efficace au vu du nombre de parutions exponentiel; le ciblage des informations littéraires devient un élément déterminant pour les internautes pour rester au plus près de l'actualité. De plus, les fils RSS rendent compte de la présence quelque part sur le net d'une information susceptible d'être récupérée sur un site pour y être diffusée et consultée par les internautes. Ceux-ci ont la possibilité de rendre disponible une partie d'un site sur un autre, ce qui participe à repérer et à valoriser les sources d'informations littéraires comme les catalogues d'éditeurs, les salons du livre, les séances de dédicaces, ou les interviews vidéo des auteurs. Ce faisant, le principe de syndication représente un outil de mutualisation de l'information dans un monde numérique submergé par le nombre de contenus disponibles.

De plus, il ne faut pas oublier que la critique se construit et s'identifie aux yeux des lecteurs comme une forme sociale porteuse d'un genre spécifique. Notre souci a été de savoir ce que devient ce genre littéraire en prenant la forme du blog. L'usage de celui-ci par les acteurs a montré une rupture volontaire. En fait, la critique sur les blogs se construit comme une forme socio-technique particulière. Les blogs permettent la mise en place d'une critique de la critique littéraire tout en proposant une alternative à des modèles de textes traditionnels. Sur Le Matricule des Anges, le blog du rédacteur en chef s'ajoute, sur le plan éditorial, au site de la revue. Nous pouvons supposer que l'utilisation de ce nouvel outil de publication en ligne correspond à la volonté d'instaurer des marques 
de reconnaissance de la fonction qu'il exerce au sein de la rédaction éditoriale du site. Le dispositif éditorial de La Revue des Ressources, par contre, est un CMS, c'est-à-dire un système de gestion de contenus $^{11}$. Il permet à ses créateurs de personnaliser la présentation du site, de limiter l'accès aux archives à un groupe d'individus, ou de proposer l'écriture de commentaires dans certaines rubriques. Quant à Zazieweb, l'indexation des homeblogs par le principe des fils RSS fait du site une communauté de electeurs. Grâce à un agrégateur de contenu, c'est-à-dire un outil logiciel permettant de recevoir des informations en temps réel provenant en même temps de plusieurs sites et blogs, les internautes ont un accès à un portail de blogs personnels exclusivement tournés vers la littérature. Nous voyons donc apparaître l'établissement d'une « relation entre [le] support de publication et [la] prescription d'usage " grâce à "l'encastrement de l'architecture éditoriale et des pratiques d'autoproduction » (Cardon et alii, $2006: 11-12$ ).

13 Toutefois, si pour les professionnels de Le Matricule des Anges et de La Revue des Ressources, il s'agit de se saisir d'un nouveau dispositif d'écriture permettant de renouveler les formats de publication traditionnels, ce n'est pas forcément vrai pour les amateurs ( Zazieweb). Leur travail d'écriture apparaît comme une activité personnalisée et interactive. Elle n'a pas pour but, comme celle des professionnels, d'asseoir leur autorité sur le web, mais d'établir une relation littéraire entre les membres d'une même communauté. A travers l'établissement des blogs littéraires, émergent des formes d'expression ordinaires à l'initiative de nouveaux publics. Nous postulons cependant que ces formes d'expression ne se limitent pas aux textes. Elles concernent aussi l'image, le son et la vidéo.

\section{L'utilisation des médias d'expression, de la représentation de soi à la consommation culturelle de la littérature}

14 Le changement observé dans l'écriture de la médiation ne touche pas seulement l'organisation des contenus des sites et les formes de la critique littéraire; elle concerne également le type de médias utilisés et l'usage qu'en font les internautes comme en témoignent de nombreux travaux effectués sur les dispositifs d'écriture en ligne dans le domaine littéraire (Chapelain et Loneux, 2007, Jeanneret, 2005, Bouchardon, 2007). Plus largement, nous rejoignons l'idée développée par P. Flichy selon laquelle le principe de création et de circulation des médias d'expression participe de l'idéologie d'une culture à un moment donné dans l'histoire des techniques (Flichy, 2001). Nous avons donc interrogé les conditions d'appropriation des nouveaux cadres intersémiotiques rendus possibles par le réseau. Nous avons mis en évidence que l'utilisation des différents médias d'expression sur les sites renouvelle la construction de l'image de soi en favorisant les pratiques de consommation des nouvelles « technologies de soi " (Foucault, 1984) sur le web.

Précisément, en s'inscrivant à la fois dans un processus de traitement de l'information et dans un contexte d'expression subjective (Fondin, 1998), le travail d'écriture littéraire sur le net devient l'expression de la subjectivité dans un monde où les acteurs sont multiples et l'effet de surproduction, en retour, favorable à l'émergence des individualités. En même temps, la mise en scène de soi convoque des logiques de mises en écran qui mettent en avant la publicisation du sujet par le biais de l'utilisation d'Internet. Le réseau 
fonctionne en effet comme un espace et un dispositif «transitionnel» (Winnicott, 1969), mais aussi comme un espace "hétérotopique» (Hert, 1999). A cet égard, si nous considérons cet espace de publicisation comme «une forme» et comme "un événement » (Quéré, 1995), la construction de l'image de soi et sa représentation, sur les sites étudiés, servent au processus de production d'une identité réflexive publique. se construit dorénavant grâce à l'image et au son, par l'installation de systèmes rich media . La présence des vidéos sur les sites montre la figure expressive de l'auteur dans son rapport avec la littérature. C'est de cette façon par exemple que les internautes du Matricule des Anges ${ }^{12}$ s'approprient les informations littéraires : en visionnant une vidéo dans laquelle l'auteur est mis en scène dans l'acte de lecture, pour promouvoir ses œuvres ou celles d'autres auteurs publiés sur le site. Au sujet de la culture de soi, B. Lahire (2004), conforte justement cette idée que les pratiques culturelles deviennent légitimes grâce à l'utilisation des nouvelles technologies. Les internautes peuvent consulter, choisir, sélectionner et utiliser les informations circulant sur le web pour leur propre expression. Ils peuvent le faire dans un espace personnel comme c'est le cas avec la réception des fils RSS audio et vidéo sur La Revue des Ressources ou sur le réseau par exemple, pour la consultation des homeblogs sur Zazieweb. Nous avons un commentaire de ces pratiques avec les propos de Robin Hunzinger, responsable du site La Revue des Ressources. Il explique que la refonte du site a démultiplié les potentialités logicielles et éditoriales de la diffusion, de la circulation et de la réception des contenus. Internet permet aussi aux internautes de s'approprier plus facilement et avec plus de liberté les informations, avec la possibilité de les réinvestir ultérieurement ${ }^{13}$.

Par ailleurs, le monde de la création numérique et multimédia, en s'invitant dans le contexte de la médiation littéraire sur le web, bouleverse l'«image du texte» de la littérature. En fait, les outils logiciels de son, audio et vidéo diversifient au maximum les formats sémiotiques. Ils participent au processus de « redocumentarisation », c'est-à-dire, pour le dire très vite, à la possibilité pour les internautes d'agencer, de combiner, de mixer les différents médias d'expression pour mettre en scène la littérature sur Internet (Pédauque, 2006). Cela rejoint encore les dires de Robin Hunzinger au sujet du site qu'il a créé : «[...] La revue des ressources, grâce au web 2.0, peut se métamorphoser et devenir un objet transmédiatique, quelque part entre littérature, arts plastiques, cinéma et radio $»^{14}$. En fait, nous retiendrons de ces propos que l'utilisation des différents médias d'expression transforme la médiation de la littérature sur le net. Il faut voir qu'au-delà des informations proposées sur les œuvres et les auteurs, les nouvelles formes expressives et médiatiques diversifient les pratiques d'écriture de la médiation littéraire sur Internet. Les formes et les outils éditoriaux rendent compte de la façonnabilité de la littérature. La médiation littéraire ne s'organise plus seulement autour d'une expression écrite débattant de la notion de littérature. Elle se crée aussi à l'aide de médias d'expression porteurs d'une communication visuelle et auditive faisant place à la consommation culturelle des nouvelles technologies.

\section{Conclusion}

Dans cet article, nous n'avons pas, certes, abordé tous les éléments participant à la construction de la médiation littéraire sur le net. Il serait sans doute nécessaire d'aller plus loin dans l'analyse des textes eux-mêmes, notamment sur le plan des contenus 
discursifs, et principalement au niveau des blogs, car leur traitement sur le plan narratif pourrait mettre en lumière l'horizon d'attente des lecteurs. Néanmoins, nous avons essayé de mettre en évidence le changement paradigmatique qui pointait à travers l'écriture actuelle de la médiation de la littérature sur le net. Le travail empirique a montré qu'il existait une médiatisation des pratiques d'écriture. De plus, la création, la diffusion et la circulation des informations révèlent une «trivialité » de la littérature, c'est-à-dire l'usage d'outils communs et de partage pour le travail d'écriture de la médiation littéraire. Internet apporte une nouvelle visibilité à la littérature en assurant une pratique des technologies récentes liée à la consommation du livre, de la lecture et des oeuvres. En fonctionnant comme des portails informationnels et médiatiques, des dispositifs d'auto-publication et de partage de documents multimédia, les sites renouvellent l'écriture de la médiation littéraire.

\section{BIBLIOGRAPHIE}

Bouchardon, S., (dir.), (2007), Un laboratoire de littératures, littérature numérique et Internet, Paris, BPI.

Cardon, D. et alii, (2006), « Présentation », L'autopublication, Réseaux, n 136, vol. 24, Paris, Hermès, pp. 9-25.

Cavelier-Croissant, V., Rebillard, F. et Touboul, A.-L., (2004), « La promotion de biens culturels par le recours aux genres journalistiques. Le cas d'Epok, consumer magazine de la FNAC, Actes du colloque Les organisations culturelles : une communication spécifique?, Université d'Avignon, disponible en ligne : http://archivesic.ccsd.cnrs.fr/docs/00/14/49/63/PDF/Epok-ActesAvignonarchive.pdf.

Chapelain, B. et Loneux, C., (2007), « Les dispositifs d'écriture en ligne : nouvelles médiations culturelles et organisationnelles ", in : Approches culturelles en sciences de l'information et de la communication, CEGES, Lille 3, pp. 129-140.

Clément, J., (2007), « Préface : une littérature problématique », in : Un laboratoire de littératures, littérature numérique et Internet, Paris, BPI, pp. 11-20.

Cotte, D., (2007), Des médias au travail. Emprunts, transferts, métamorphoses, Habilitation à Diriger des Recherches, Université d'Avignon et des Pays du Vaucluse.

Davallon, J. et Jeanneret, Y., (2004), « La fausse évidence du lien hypertexte », Communication \& Langages, $\mathrm{n}^{\circ} 140, \mathrm{pp} .43-54$.

Davallon, J., Brochu, D. et Noël-Cadet, N., (2003), «L'usage dans le texte : les 'traces d'usages' du site Gallica », in : Souchier E., Jeanneret Y. et Le Marec J. (dir.), Lire, écrire, récrire : objets, signes et pratiques des médias informatisés, Paris, BPI, pp. 47-90.

Jeanneret, Y., (dir.), (2005), Métamorphoses médiatiques, pratiques d'écriture et médiation des savoirs, Rapport final de recherche, ACI cognitique, Programme « société de l'information », CNRS, disponible en ligne : http://www.lalic.paris4.sorbonne.fr/metamorphose/source/ rapport_final.pdf. 
Jeanneret, Y. et Souchier, E., (2005), «L'énonciation éditoriale dans les écrits d'écran », Communication \& Langages, $\mathrm{n}^{\circ} 145$, pp. 3-15.

Flichy, P., (2001), L'imaginaire d'Internet, Paris, La Découverte.

Fondin, H., (1998), Le traitement numérique des documents, Paris, Hermès.

Foucault, M., (1984), Dits et écrits, Paris, Gallimard.

Hert, P., (1999), « Internet comme dispositif hétérotopique », Hermès, n² 25, pp. 93-107.

Lahire, B., (2004), La culture des individus - dissonances culturelles et distinctions de soi, Paris, La Découverte.

Pédauque, R., (2006), Document et modernités, disponible en ligne : http://archivesic.ccsd.cnrs.fr/ docs/00/06/28/26/PDF/Pedauque3-V4.pdf.

Quéré, L., (1995), « L'espace public comme forme et comme événement », in : Joseph I., (textes réunis par), Prendre place. Espace public et culture dramatique, Éditions Recherches, pp. 93-101.

Souchier, E., Jeanneret, Y. et Le Marec, J., (dir.), (2003), Lire, écrire, récrire : objets, signes et pratiques des médias informatisés, Paris, BPI.

Stockinger, P., (2005), Les sites web : conception, description et évaluation, Paris, Hermès.

Winnicott, D. W., (1969), Jeu et réalité. L'espace potentiel, Paris, Gallimard.

\section{NOTES}

1. Employée dans notre travail de thèse pour l'analyse de vingt-six sites de littérature de jeunesse, cette approche a eu pour but de cerner les stratégies éditoriales et communicationnelles que les acteurs culturels et pédagogiques mettent en place sur le net pour diffuser les informations relatives aux livres pour enfants. Cf. K. Soumagnac, La littérature de jeunesse en ligne, médiation et pratiques médiatiques, thèse de doctorat en sciences de l'information et de la communication, Université de Lille 3, 2006, 392 p.

2. Adresse du site http://www.zazieweb.fr. Créé en 1996, la fondatrice du site Isabelle Aveline définit Zazieweb comme étant la communauté des e-lecteurs.

3. Adresse du site http://www.larevuedesressources.org. La Revue des Ressources est une revue en ligne créée en 1998 par Robin Hunzinger et Bernard Gauthier. Le site littéraire du même nom représente pour les auteurs « une expérience de revue permanente, régulièrement mise à jour ». Pour plus d'informations concernant la création du site, il est possible de se reporter à la page dont est issue la citation ci-dessus: http://www.larevuedesressources.org/article.php3? id_article $=115$.

4. Adresse du site http://www.lmda.net. Créé en octobre 1992, Le Matricule des Anges est un magazine indépendant d'informations littéraires diffusé à 7000 exemplaires sur toute la France.

5. Zazieweb propose des liens vers les émissions littéraires de France Culture (Mauvais Genre), de France 5 (Le bateau-Livre), de France 2 (Des mots de Minuit), Arte (Metropolis) pour n'en citer que quelques-uns.

6. Sur Le Matricule des Anges, les librairies virtuelles comme chapitre.com, abebooks.fr ou encore lelibraire.com sont accessibles dès la page d'accueil et à l'intérieur de toutes les rubriques du site. 7. Les trois sites, dans leur espace publicitaire, renvoient à des sites en lien avec la publication et l'édition d'écrits comme par exemple http://www.manuscrit.com, http://www.edilivre.com, http://www.societedesecrivains.com, http://www.jepublie.com, etc. 
8. Le site Le Matricule des Anges a créé un blog pour les Présidentielles de 2007 consultable à l'adresse suivante: http://www.lmda.net:16080/blog. Ce blog n'est plus accessible depuis octobre 2007 à partir du site.

9. Les fils RSS sont une famille de formats XML utilisés pour la syndication de contenus sur le web. Les fils permettent d'obtenir les mises à jour d'information sur les sites ou les blogs.

10. Voir les travaux spécifiques sur ces nouveaux outils de veille documentaire. Des études sur la mise en place de cet outil ont été effectuées dans le domaine de la veille stratégique au niveau des entreprises. On peut se reporter notamment à l'article de Olivier Erztscheid intitulé «Weblogs: un nouveau paradigme pour les systèmes d'information et la diffusion des connaissances?» à consulter à la page suivante: http://archivesic.ccsd.cnrs.fr/ docs/00/06/26/19/PDF/sic_00001433.pdf.

11. Les systèmes de gestion de contenus sont des logiciels de gestion et de mise à jour dynamique des sites web ou d'applications multimédia. Ils permettent notamment à plusieurs individus de travailler sur un même document, de fournir une chaîne de publication pour mettre en ligne des contenus documentaires, de séparer les opérations de gestion de la forme et du contenu, et de structurer le contenu.

12. Les vidéos sont téléchargeables à l'adresse suivante: http://www.lmda.net/direct/ video.html.

13. «[...] La refonte a permis de mettre en place de nouveaux éléments afin de répondre aux attentes des lecteurs et de leur offrir un site plus fonctionnel et attractif. La revue en elle-même reste le vecteur des recherches et des expérimentations de ses auteurs [...] ». Propos de Robin Hunzinger relevés dans l'éditorial de la revue du jeudi 14 septembre 2006 à l'adresse suivante : http://www.larevuedesressources.org/article.php3?id_article=638.

14. Ces propos de Robin Hunzinger sont consultables à la même adresse que celle indiquée à la note précédente : http://www.larevuedesressources.org/article.php3?id_article=638.

\section{RÉSUMÉS}

Les outils du web 2.0, en modifiant les pratiques éditoriales traditionnelles, transforment peu à peu la médiation de la littérature sur le net. Pour comprendre les mutations à l'œuvre, nous avons abordé les écrits d'écran littéraires par une approche "techno-socio-sémiotique » des formes d'écriture. Les changements observés interviennent à plusieurs niveaux. Tout d'abord, les liens hypertexte orientent l'attention des usagers vers les rubriques d'actualités littéraires des sites des médias traditionnels. Ensuite, l'organisation des contenus implique la participation d'acteurs multiples et un travail d'écriture collaboratif. En outre, les blogs renouvellent la critique littéraire en proposant de nouvelles formes d'expression ordinaires. En dernier lieu, l'utilisation des médias expressifs comme le son, l'image et la vidéo sur les sites, reflète des pratiques socio-techniques qui montrent la mise en scène de l'internaute et le caractère composite et médiatique de la littérature dans l'espace public numérique.

By modifying traditional editorial practices, Web 2.0 tools are gradually transforming literary mediation on the Internet. To understand the changes at work, we address on-screen literary writing from a techno-socio-semiotic approach to writing. The changes observed occur at several levels. Firstly, hypertextual links guide the users' attention to current literary contributions on the websites of traditional media. Secondly, the organization of the content implies the 
participation of numerous actors and a collaborative form of writing. In addition, blogs renew literary reviews by proposing new varieties of ordinary expressions. Finally, the use of such media of expression on websites as sound, images, and video reflects socio-technical practices that reveal the act of production by Internet users and the composite and mediatized character of literature in public cyberspace.

\section{INDEX}

Mots-clés : médiation, internet, littérature, pratique éditoriale, organisation du contenu, média d'expression, écriture, lien hypertexte, blog

Keywords : internet, mediation of literature, writing practices, organisation of information, media of expression, writing, hypertext link, blog

\section{AUTEUR}

\section{KAREL SOUMAGNAC}

Karel Soumagnac est docteure en sciences de l'information et de la communication et chargée de cours à l'université Charles-de-Gaulle - Lille 3. Chercheure associée au laboratoire GERIICO, ses recherches portent principalement sur le rapport entre la médiatisation des nouvelles formes d'écriture et les pratiques de consommation culturelles sur Internet à propos de la littérature. 